\title{
A Delayed Presentation of an Extrapleural Hematoma After a Blunt Chest Trauma in an Anticoagulated Patient
}

\author{
Claudio Bianchin ${ }^{1 *}$, Carolina Prevaldi ${ }^{2}$, Matteo Corradin ${ }^{3}$, Mario Saia ${ }^{4}$, Paolo D’ Andrea ${ }^{5}$ and Manuela Crucitti ${ }^{6}$ \\ ${ }^{1}$ Local Health Unit no.1, Department of Risk Management and Patient's Safety, S. Martino Hospital, Belluno, Italy
}

${ }^{2}$ Local Health Unit no 4, Department of Risk Management and Patient's Safety, Italy

${ }^{3}$ Regional Center for Patient Safety, Italy

${ }^{4}$ Medical directorate, Local Health Unit of Padua no. 6, Italy

${ }^{5}$ Department of Radiology, S. Martino Hospital, Belluno, Italy

${ }^{6}$ Department of Anethesiology, S. Martino Hospital, Belluno, Italy

Submission: July 27, 2018; Published: August 09, 2018

*Corresponding author: Claudio Bianchin, Servizio Medicina Legale ULSS 1, S. Martino Hospital, 32100 Belluno, Italy, Tel: +39.0437.514233;

Email: claudio.bianchin@ulss.belluno.it

\begin{abstract}
We describe a case of a voluminous Extra Pleural Haematoma (EPH), occurred the day after a vehicular accident in an anticoagulated male patient for atrial fibrillation. A full body Computed Tomography (CT) showed multiple rib fractures and a posterior extra pleural opacity, associated with a partial atelectasis of the right lower lobe. Carefully monitored, the patient was in a stable hemodynamic and respiratory state, in a first time. However, due to a faster mass expansion, he started deteriorating abruptly. The patient underwent an emergency chest drain positioning, when shortly a cardiac arrest occurred due to a mediastinum shift on the left -hand side. As a result, he suffered from a severe brain
\end{abstract} damage. Here, based on the case, we discuss diagnostic and therapeutic problems of extra pleural hematoma.

\section{Introduction}

Extra pleural hematoma is a rare and dangerous condition which can occur as a result of a chest trauma with multiple rib fractures due to an intercostal vessels tear. A delayed diagnosis and treatment might have serious cardiovascular and respiratory consequences and, not unusual, can lead to die. It is characterized by a pool of blood between parietal pleura and the endothoracic fascia. Anticoagulated patients are particularly at risk. Chest pain, dyspnoea and/or signs and symptoms of anaemia and hypovolemia typically characterize symptoms. The diagnosis can be often difficult and delayed, needing to be confirmed in any case by a CT scan with intravenous (i.v.) contrast. The management can be just conservative. Sometimes it is inevitable to insert a tube thoracostomy or perform a thoracoscopy (or thoracotomy) to repair damaged vessels. Embolization of bleeding is also possible. This case illustrates an extra pleural hematoma developed after a thoracic trauma following a vehicle accident in a patient taking anticoagulation therapy.

\section{Case Report}

An 86 years old man with a past medical history of atrial fibrillation on anticoagulant, was brought by ambulance in Emergency Department (ED), following a vehicle accident. A full body CT scan with contrast did not show traumatic injuries, however he was taken under close observation in recovery area. He was stable overnight, and he was discharged the morning after, making a diagnosis of multiple contusions. His International Normalized Ratio (INR) was 3.20. At night, he returned to the hospital, complaining of dyspnoea. A new CT scan was obtained, which revealed a $16-\mathrm{cm}$, right- sited, posteriorly localized pool of blood, likely to be extra pleural (Figure 1).

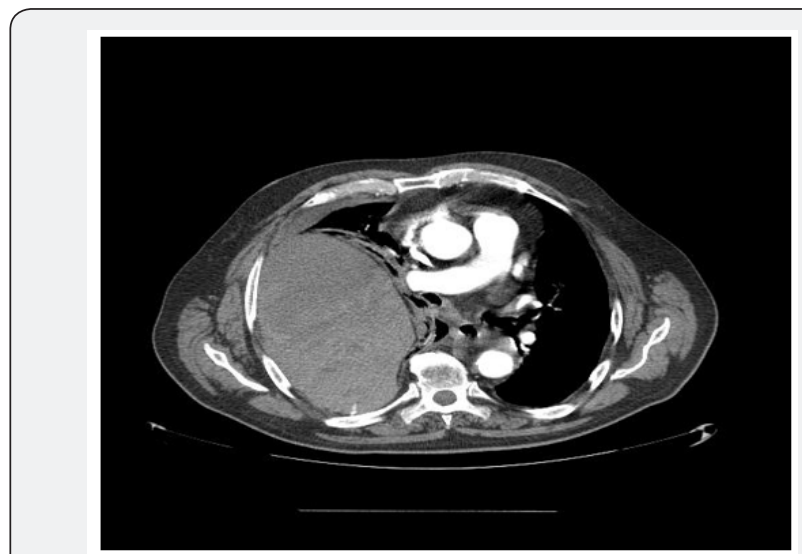

Figure 1: CT scan right- sited extra pleural hematoma. 
A partial atelectasis of the right lower lobe and multiple posterior rib fractures were associated. On physical examination, oxygen saturation was $91 \%$ while he was breathing ambient air, blood pressure $120 / 80 \mathrm{mmHg}$, heart rate 118 beats per minute. Haemoglobin was $15.1 \mathrm{~g} / \mathrm{dl}$. The patient was subsequently transferred to the surgical ward. He was constantly monitored overnight. Although he looked like restless and stricken, he was haemodynamically and respiratory stable, keeping a good oxygen saturation. In the morning, he started deteriorating. He was diaphoretic and cyanotic. A third contrast-enhanced CT of the chest revealed a significant increase of the lesion measuring $240 \mathrm{x}$ $180 \mathrm{~mm}$. This was accompanied by a mediastinum slightly shifted on the left-hand side. Haemoglobin was $11.5 \mathrm{~g} / \mathrm{dL}$ at this point, and fresh frozen plasma was required. A decision to bring the patient to theatre and perform a thoracotomy was promptly made and evacuation of $1000 \mathrm{ml}$ of blood was performed. Meanwhile he went to cardiac arrest. He was resuscitated, but he reported a detrimental brain damage followed by a persistent vegetative state.

\section{Discussion}

Extrapleural haematoma is a rare occurrence, sparsely reported in the literature as a case reports or series, which is caused by injury to intercostal arteries or veins after high-energy blunt trauma, especially those involving rib fractures. Hemo thorax, pneumothorax and lung contusions may also occur in EPH cases as well as extra thoracic lesions [1,2]. Though, a delayed diagnosis and treatment are also strongly associated with a hemodynamic and respiratory instability and high mortality rate. EPH is characterized by the accumulation of blood in the extra pleural space, between the parietal pleura and thoracic fascia. Patients taking anticoagulant drugs are particularly at risk because of an elevated INR and impaired clot formation. Consequently, those patients bleed much more in a little period of time [3-5].

Some pre-existing pleural synechiae can be a contributory cause. In many cases EPH and its symptoms may occur several hours or days after trauma. Rib fractures can cause damage by crushing arteries and veins even during dressing or daily routine, especially in the elderly. Symptoms are typically characterized by chest pain, dyspnoea, due to a progressive lung's compression and/or signs and symptoms of anaemia and hypovolemia (hypotension, tachycardia, tachypnoea, agitation, paleness). EPH and coexistence of rib fractures may be hypothesized, often in late, on the basis of radiographic findings. Nevertheless, it is mandatory a chest CT scan with contrast to confirm the diagnosis [6].

At the beginning, in some recurrent cases with a latent stage, radiological investigations might not show anything, as a bleeding or an intrathoracic mass [7]. Although the hematoma can be spontaneously re-absorbed, sometimes the growth can lead to a mediastinum shift and impaired venous return. If rupture occurs blood can flow into pleural space, causing haemothorax and severe anaemia [8]. Treatment of a small EPH, with spontaneous regression, can be easily managed conservatively, with fluid integration or blood transfusions. If a large EPH causes respiratory and circulatory disturbances, evaluation may be required, by performing a thoracostomy.

A thoracotomy and/or video-assisted thoracoscopy are useful to identify and repair blood vessels, as well as embolization is a possible treatment. Embolization can be selective or superselective. It is indicated when the source of bleeding is not identified or in cases which comorbidities are uncountable or there are significant coexisting lesions and contraindications to surgery. In an anticoagulated, bleeding patient who needs an intervention, anticoagulation reversal with Prothrombin Complex Concentrates (PCCs) rapidly restores haemostasis, but, on the other side, the thrombotic risk due to their procoagulant effect should be considered [9-12]. A careful monitoring of hemodynamic and respiratory parameters is appropriate as well as a series of CT to verify the evolution of the lesion. If the patient shows hemodynamic instability a prompt surgical intervention is mandatory.

\section{References}

1. MA Rashid, T Wikstrom, P Ortenwall (2000) Nomenclature, classification, and signature of traumatica extrapleural hematoma. The journal of trauma 49(2): 286-290.

2. AS Bolliger, MJ Thali, E Aghayev, C Jackowski, P Vock, et al. (2007) Postmortem noninvasive virtual autopsy - extrapleural hemorrhage after blunt thoracic trauma. Am J of Forensic Med Pathol 28(1): 44-47.

3. Mingoli, M Assenza, R Petroni, A Antoniozzi, G Branchini, et al. (2004) Large extra pleural hematoma in an anticoagulant patient after a thoracic blunt trauma. Ann Ital Chir 75(1): 83-85.

4. HO Sumida, N Ono, Y Terada (2007) Huge extra pleural hematoma in an anticoagulated patient. Gen Thor Cardiovasc vSurgery 55(4): 174176.

5. H Igai, N Okumura, K Ohata, T Matsuok, K Kameyama, et al. (2008) Rapidly expanding extra pleural hematoma. Gen Thor Cardiovasc Surgery 56(10): 515-517.

6. SL Aquino, C Chiles, T Oaks (1997) Displaced extra pleural fat revealed by CT scanning: evidence of extra pleural hematoma. AJR Am J Roentgenol 169(3): 687-689.

7. EH Carrillo, BT Heniford, SO Senler, JR Dykes, SP Maniscalco, et al. (1998) Embolisation therapy as an alternative to thoracotomy in vascular injuries of the chest wall. Am Surg 64: 1142-1148.

8. Reiter C, Denk W (1985) Delayed hematoma as a fatal complication following blunt chest injury. Wiener Klinische Wochenschrift 97(12): 535-537.

9. AP Chemelli, M Thauerer, F Wiedermann, A Strasak, J Klocker, et al. (2009) Transcatheter arterial embolisation for the management of iatrogenic and blunt traumatic intercostal artery injuries. J Vasc Surg 49(6): 1505-1513.

10. AS Poyraz, D Kilic, B Gultekin, M Ozulku, A Hatipoglu (2005) Extrapleural hematoma: when is surgery indicated? Monaldi Arch Chest Dis 63(3): 166-169.

11. B Annu, K Vishal, S Sushma, K Subodh, G Amit (2015) Large extrapleural hematoma: a rare delayed presentation after blunt toracic trauma. J Trauma Treat 4: 241.

12. MG Pirzirenli, B Clik, S Gurz, ZP Surucu (2015) Extra pleural hematoma case treated wih video- assietd thoraccoscopi surgery. Ulus Trauma Acil Cerrahi Derg 21(5): 405-409. 


\section{Your next submission with Juniper Publishers} will reach you the below assets

- Quality Editorial service

- Swift Peer Review

- Reprints availability

- E-prints Service

- Manuscript Podcast for convenient understanding

- Global attainment for your research

- Manuscript accessibility in different formats

( Pdf, E-pub, Full Text, Audio)

- Unceasing customer service

Track the below URL for one-step submission https://juniperpublishers.com/online-submission.php 